\title{
A Penalty-based Approach for Contact Forces Computation in Bipedal Robots
}

\author{
Carlos Rengifo, Yannick Aoustin, Christine Chevallereau and Franck Plestan \\ IRCCyN, Ecole Centrale de Nantes-Université de Nantes CNRS, Nantes, France \\ \{prenom.nom\}@irccyn.ec-nantes.fr
}

\begin{abstract}
Computation of contact forces is essential for the simulation of mechanical systems with unilateral constraints, like bipedal robots. Most methods are based on the rigid body assumption. They can be categorized into constraint-based and penalty-based approaches. In the former, contact forces are computed by solving an optimization problem based on linear or nonlinear complementarity conditions. Unfortunately, these methods cannot be directly applied to articulated systems described in generalized coordinates. In the second approach, spring-damper models are used to minimize interpenetration between the surfaces in contact. The main criticism to penalty approaches are parameter tunning, static friction handling, and the difficulties to treat multiple simultaneous unilateral contacts. In this work we present a new compliant approach based on input-output feedback linearization. The main advantages of the proposed approach are, the spring-damper parameters are independent of the parameters of the system (i.e masses, inertias), no a priori-defined velocity thresholds are required to distinguish between dynamic and static friction, multiple simultaneous unilateral contacts are naturally handled. The proposition has been succesfully applied to the simulation of a 3D bipedal walking robot.
\end{abstract}

\section{INTRODUCTION}

Bipedal robots are mechanical systems with contacts intermittence, unilateral constraints and impacts. These systems are traditionally described as hybrid dynamical systems and by consequence biped walking simulators commonly uses an event-driven approach: impacts are regarded as the events producing transitions between biped phases. In the case of bipedal robots with point-feet there are only four possible phases: right single support, left single support, double support and fly. In robots with no trivial feet the number of states and events dramatically increases. For example, in a rectangular footed biped, each foot may be contacting in one of 10 ways (at one of four vertices, one of four edges, with its face, or not in contact at all) and the foot can impact the ground in 9 different ways. Thus, the resulting system is a finite state machine with 100 states and 81 possibles events. In [6], as contacts with static friction are handled separately from those with dynamic friction, their software platform for humanoid robot simulation considers 361 possible contact states. The contact enumeration problem is discussed in [15]. Given the exploding complexity of the event-driven approach as the number of contact points increases, techniques stemmed from computer graphics animations are currently applied to humanoid robot simulators. These techniques can be categorized into constraint-based and penalty-based approaches. In the former, contact forces are computed by solving an optimization problem based on linear or nonlinear complementarity conditions. The most natural way to formulate these conditions is in terms of accelerations and forces. However it leads to problems without solution in the classical sense. Even with the use of impulsive forces, the existence of solutions can not be guaranteed [14]. Instead of acceleration and forces, complementarity conditions can be formulated in terms of velocities and time integrals of the forces, provided the transformation of the differential equations of the system into finite differences. Many variants of this approach, called time-stepping, have been proposed. Anitescu [1] and Stewart [12] use a linear complementarity formulation requiring the approximation of the friction cone by a polyhedron. The advantage of Anitescu and Stewart methods is the existence and uniqueness of solutions can be guaranteed [13]. Unfortunately, these methods are formulated using maximal coordinates and by consequence cannot be directly applied to articulated systems described in generalized coordinates. When maximal coordinates are used, joints must be treated as bilateral constraints. The major disadvantage of this is interpenetration due to numerical drift. Interpenetration must be corrected using post stabilization methods like [4]. The proposition of Liu and Wang [9] uses maximal coordinates also, but it does not require some friction cone approximation. However, bilateral constraints are not considered, so its applicability to robotic systems is very limited. All timestepping approaches have the advantage that collisions and contact forces are computed in a unified way. On the other hand, they have many limitations from the computational point of view as stated in [5], and they do not have been extended to articulated bodies described with generalized coordinates. In [3] a simulator for the robot HRP2 using a combination of time-stepping and the acceleration-force formulation proposed in [11] is presented.

In the penalty based approach spring-damper models are used to minimize interpenetration between the surfaces in contact [16]. Penalty methods are used in most biped simulators. Some recent examples are Saika-3 [8], JOHNNIE [2] and OpenHRP [7]. In these biped simulators spring-damper models are used to compute normal and tangential contact forces. In [8], a classical spring-damper model is used, the problem is that sticking normal forces appear just before 
contact separation. In [2], a Heaviside function is used to prevent this problem. In [7] the computation of normal contact forces is based on the nonlinear spring-damper model proposed in [10]. This model assures continuous normal contact forces. The problem is that sticking forces arise when an external force separates the objects at high velocity. That is the case in biped walking simulations when the stance foot takes off after the impact. The three above mentioned methods do not require velocity thresholds to distinguish between static and dynamic friction. The main criticisms to these penalty-based approaches are parameter tunning, and the difficulties to treat multiple simultaneous unilateral contacts.

In this paper a new penalty-based approach based on inputoutput feedback linearization is proposed. The main advantages of the proposed approach are: the parameters of the contact model are independent of those of the system (i.e masses, inertias), no a priori-defined velocity threshold are required to distinguish between dynamic and static friction and multiple simultaneous unilateral contacts are naturally handled. This paper is organized as follows. Section II describes the proposed methodology for the contact force computation using two simple examples. In section III the methodology is applied to a bipedal robot with rectangular feet. In Section V, the numerical problem of contact stability is introduced. In Section IV simulation results are presented. The last section of this paper is devoted to conclusions and perspectives.

\section{THE PROPOSED APPROACH}

In order to illustrate the general idea of the proposed methodology, two simple examples are considered. The first one is devoted to the normal force computation, and the second one to friction forces. The incidence of the contact model parameters on the resulting reaction forces will be also illustrated.

\section{A. Bouncing ball}

Consider a bouncing ball of mass $m$ as illustrated in Figure 1. The differential equation for the position is:

$$
m \ddot{p}_{z}=-m g+f_{n}
$$

The normal contact force $f_{n}$ is zero when the position $p_{z}$ is positive, and $f_{n}$ is greater than zero when $p_{z}=0$. If we want to simulate the system (1) then raises the question how to compute the reaction force between the ball and the ground?. If a rigid impact with restitution coefficient zero is supposed, then the resulting $f_{n}$ has two components as illustrated in Figure 2. A Dirac's delta function of weight $-m \dot{p}_{z}\left(t_{i}^{-}\right)$; and constant value $m g$ for $t>t_{i}$. The impulse, which occurs at the impact instant $t=t_{i}$, is required to discontinuously change the velocity from a negative value to zero. The constant component of $f_{n}$ guarantees $\ddot{p}_{z}\left(t>t_{i}\right)=0$. The function $f_{n}$ of the Figure 2 assures zero velocity and acceleration after the impact. This example clearly shows the

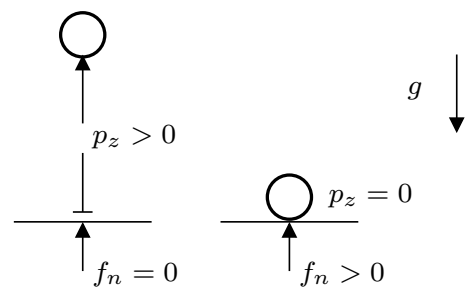

Fig. 1. Bouncing ball

separation between impact and resting contact forces. In the penalty methods used in the simulators for the bipedal

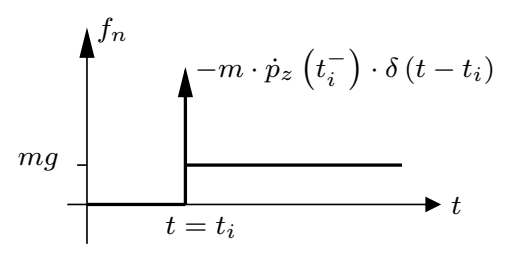

Fig. 2. Theoretical reaction force for the bouncing ball example when a rigid impact with zero restitution coefficient is supposed.

robots Saika-3 [8], Johnnie [2] and OpenHRP [7], impact and resting contact forces are computed in a unified way. When a typical penalty-based method like [8], is applied to the bouncing ball system, $f_{n}$ is computed using a springdamper model:

$$
f_{n}=\left\{\begin{array}{cc}
0 & p_{z}>0 \\
-k_{p} p_{z}-k_{v} \dot{p}_{z} & p_{z} \leq 0
\end{array}\right.
$$

As noted in [10], the main inconvenient of expressions like (2), is that before contact separation forces tending to stick the ball to the ground always appear. The expressions used in the biped simulators described in [7] and [2], avoid this problem using nonlinear functions.

In the approach proposed in this paper, when $p_{z} \leq 0$, we consider that the acceleration of the ball is imposed by the contact model. This acceleration is opposed to interpenetration

$$
\ddot{p}_{z}^{d}=-k_{p} p_{z}-k_{v} \min \left(0, \dot{p}_{z}\right)
$$

Hereafter $\ddot{p}_{z}^{d}$ will be called the desired acceleration. If $k_{p}>0$ and $k_{v}>0$, the term $\min \left(0, \dot{p}_{z}\right)$ assures a desired positive acceleration for all $p_{z}<0$. Once we have $\ddot{p}_{z}^{d}$, the following step consists to compute the required $f_{n}$ to obtain $\ddot{p}_{z}=\ddot{p}_{z}^{d}$. For the model (1) such force is $f_{n}=m g+m \ddot{p}_{z}^{d} . f_{n}$ in the general case is given by

$$
f_{n}=\left\{\begin{array}{cc}
0 & p_{z}>0 \\
m g+m \ddot{p}_{z}^{d} & p_{z} \leq 0
\end{array}\right.
$$

The procedure for the computation of $f_{n}$ when $p_{z} \leq 0$ is summarized in Figure 3: first, the desired acceleration is computed using the equation (3) and then the corresponding contact force is computed using (4). In the general case a 
saturation function is applied to assure $f_{n} \geq 0$. In this simple case $\ddot{p}_{z}^{d} \geq 0$ implies $f_{n} \geq 0$. As the equation (3) can be rewritten as

$$
\ddot{p}_{z}^{d}=k_{p}\left(p_{z}^{d}-p_{z}\right)+k_{v} \max \left(0, \dot{p}_{z}^{d}-\dot{p}_{z}\right)
$$

$f_{n}$ is the required force to obtain a desired position $p_{z}^{d}=0$ and a desired velocity $\dot{p}_{z}^{d}=0$. A fundamental difference between (2) and (4) is steady state interpenetration depth. In the former case, this value is equal to $-g . m / k_{p}$ and for the second expression is zero. However, in the Section V the case of a nonzero desired interpenetration is discussed.

Given $\dot{p}_{z}\left(t_{i}\right)<0$ and $p_{z}\left(t_{i}\right)=0$, the velocity and the position of the ball at the impact instant $t=t_{i}$, the dynamical behaviour in contact is determined by the following differential equation:

$$
\ddot{p}_{z}=-k_{p} p_{z}-k_{v} \min \left(0, \dot{p}_{z}\right), \quad t \geq t_{i}
$$

Parameter selection for the contact model concerns only the equation above and does not depend on the mass of the ball. In more complex systems the proposed procedure allows a parameters selection independently of the mathematical model of the systems and the number of points in contact with the ground. In Figures 4 and 5 simulation results are presented for different values of $k_{v}$ and $k_{p}$. When $k_{p} / k_{v}$ varies from 0 to $\infty$, the restitution coefficient varies from 0 to 1 . If $k_{p}=0$ and $k_{v}>0$ the interpenetration cannot vanish (Figure 4- Dashed line). In this case the penetration depth decreases as $k_{v}$ increases. If $k_{p}=0$ and $k_{v} \rightarrow \infty$ the obtained $f_{n}$ tends to that described in Figure 2. When $k_{p}>0$ interpenetration converges toward zero. When $k_{p}>0$ and $k_{v}=0$, the maximal penetration depth decreases as $k_{p}$ increases. At impact instant $f_{n}=m g-m k_{v} \dot{p}_{z}$, so the maximal value of the normal contact force depends only on $k_{v}$.

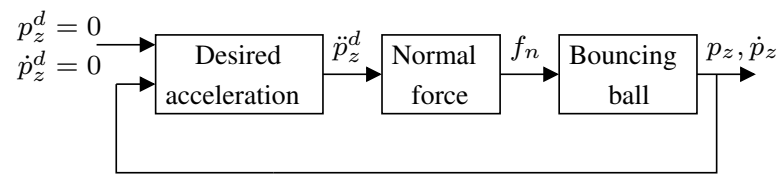

Fig. 3. Bouncing ball. Computation of the normal force when $p_{z} \leq 0$.

\section{B. Brick on a frictional ramp}

Consider a brick sliding down a ramp as illustrated in Figure 6. The differential equation for the position is:

$$
m \ddot{p_{x}}=m g \sin \alpha-f_{r}
$$

Where $f_{r}$ is the frictional force. If the initial velocity is strictly positive $\left(\dot{p}_{x}(0)>0\right)$, and the frictional force is greater than the gravitational one $(\mu \cos \alpha>\sin \alpha)$, then the velocity of the brick goes to zero and the brick will be stopped. In such case, if Coulomb's friction model is applied, $f_{r}$ is a discontinuous function of the velocity:

$$
f_{r}=\left\{\begin{array}{cc}
\mu m g \cos \alpha \operatorname{sign}\left(\dot{p}_{x}\right) & \left|\dot{p}_{x}\right|>0 \\
m g \sin \alpha & \dot{p}_{x}=0
\end{array}\right.
$$

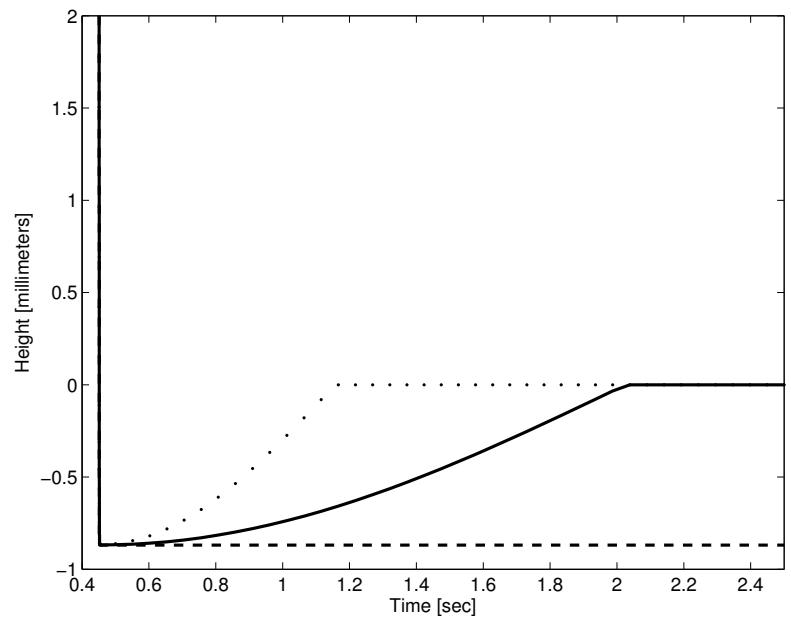

Fig. 4. Bouncing ball vertical position. Height $(\mathrm{mm})$ versus time $(\mathrm{sec})$ : $k_{p}=0, k_{v}=5000$ (dashed line), $k_{p}=1, k_{v}=5000$ (solid line), $k_{p}=5, k_{v}=5000$ (dotted line).

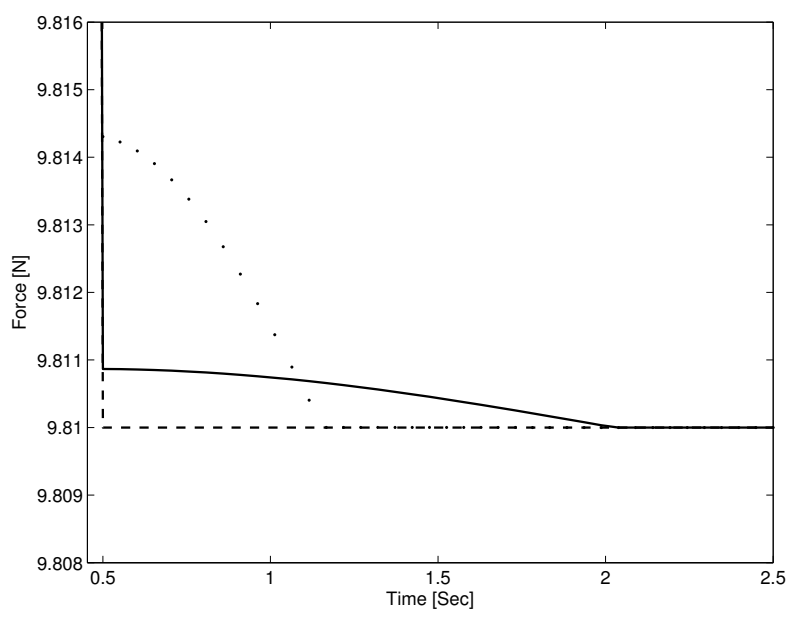

Fig. 5. Bouncing ball normal reaction force. Force $(N)$ versus time (sec): $k_{p}=0, k_{v}=5000$ (dashed line), $k_{p}=1, k_{v}=5000$ (solid line), $k_{p}=5, k_{v}=5000$ (dotted line)

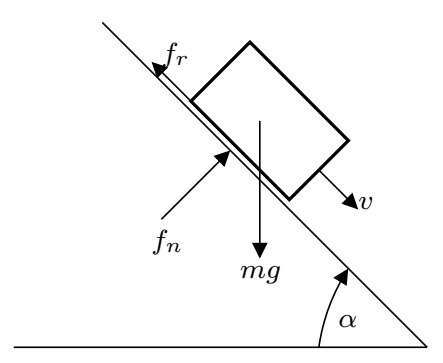

Fig. 6. Brick on a frictional ramp. 
Now we will apply a procedure similar to that of the last example. The desired tangential acceleration is chosen as:

$$
\ddot{p}_{x}^{d}=-k_{v} \dot{p}_{x}
$$

The required friction force $f_{r}$ to obtain $\ddot{p}_{x}=\ddot{p}_{x}^{d}$ from (5) is given by $f_{r}=m g \sin \alpha-m \ddot{p}_{x}^{d}$. However, the magnitude of the frictional force is limited by the normal force and the friction coefficient. Thus, a saturation function must be applied in order to assure an $f_{r}$ such that $\left|f_{r}\right| \leq \mu f_{n}$. The normal force exerted by the ramp on the brick is $f_{n}=m g \cos \alpha$

$$
f_{r}=\operatorname{sign}\left(f_{r}^{\prime}\right) \cdot \min \left(\left|f_{r}^{\prime}\right|, \mu m g \cos \alpha\right)
$$

with

$$
f_{r}^{\prime}=m g \sin \alpha+m k_{v} \dot{p}_{x}
$$

The equation (8) can be rewritten in a more classical form:

$$
f_{r}=\left\{\begin{array}{cc}
\mu m g \cos \alpha \cdot \operatorname{sign}\left(\dot{p}_{x}\right) & \left|\dot{p}_{x}\right|>v_{s} \\
m g \sin \alpha+m k_{v} \dot{p}_{x} & \left|\dot{p}_{x}\right| \leq v_{s}
\end{array}\right.
$$

With $v_{s}=\left(g / k_{v}\right)(\mu \cos \alpha-\sin \alpha)$. When $\left|\dot{p}_{x}\right| \leq v_{s}$ the model (5) becomes $\ddot{p}_{x}=-k_{v} \dot{p}_{x}$. In such case, the velocity and the force respectively converge to zero and to $m g \sin \alpha$. The procedure for the computation of $f_{r}$ is summarized in Figure 7: first, the desired acceleration is computed using the equation (7) and then the corresponding contact force is computed using (9); second a saturation function is applied to this force in order to assure $\left|f_{r}\right| \leq \mu f_{n}$. As it can be seen from the Figure $7, f_{r}$ is the required force to obtain a desired velocity $\dot{p}_{x}^{d}=0$, given the constraint $\left|f_{r}\right| \leq \mu f_{n}$.

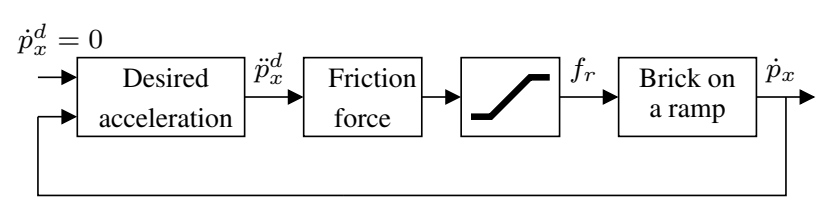

Fig. 7. Brick on frictional ramp. Computation of the friction force.

\section{COMPUTATION OF CONTACT FORCES IN A BIPEDAL ROBOT USING INPUT-OUTPUT FEEDBACK LINEARIZATION}

The bipedal robot used in this paper is depicted in Figure 8. It consists of five rigid links and two rectangular feet connected by fourteen motorized joints to form a serial structure. Eight possible contact points are considered, one for each vertice of the foot. Impacts on edges are treated as a simultaneous collision with the ground of two vertices. Flat foot impacts are treated as a simultaneous collision with the ground of four vertices. The mathematical model of the biped is given by:

$$
A(q) q_{a}+H\left(q_{p}, q_{v}\right)=\Gamma_{e}+D\left(q_{p}\right) F
$$

With

$$
q_{a}=\left[\begin{array}{c}
{ }^{0} \dot{V}_{0} \\
0 \dot{\omega}_{0} \\
\ddot{q}
\end{array}\right], \quad q_{v}=\left[\begin{array}{c}
{ }^{0} V_{0} \\
{ }^{0} \omega_{0} \\
\dot{q}
\end{array}\right]
$$

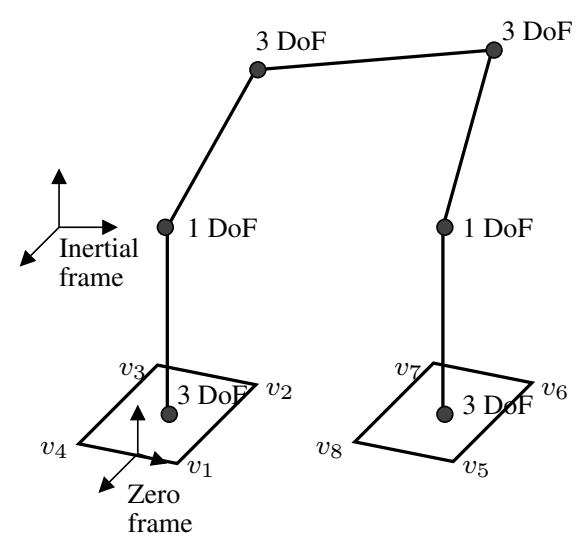

Fig. 8. General diagram of the bipedal robot and notation for the vertices.

$$
q_{p}=\left[\begin{array}{c}
X_{0} \\
\alpha \\
q
\end{array}\right], \quad \Gamma_{e}=\left[\begin{array}{l}
0 \\
0 \\
\Gamma
\end{array}\right]
$$

where $X_{0}$ and $\alpha$ are respectively the position and orientation of the right foot in the inertial frame. $q \in \mathbb{R}^{14}$ are joint positions. ${ }^{0} V_{0} \in \mathbb{R}^{3}$ and ${ }^{0} \omega_{0} \in \mathbb{R}^{3}$ are respectively the linear and angular velocity of the zero-frame. ${ }^{0} \dot{V}_{0} \in \mathbb{R}^{3}$ and ${ }^{0} \dot{\omega}_{0} \in \mathbb{R}^{3}$ are respectively the linear and angular acceleration of the zero-frame. These velocities and accelerations are defined as:

$$
\begin{aligned}
{ }^{0} V_{0} & \equiv{ }^{0} R_{I} \cdot{ }^{I} V_{0} & { }^{0} \omega_{0} & \equiv{ }^{0} R_{I} \cdot{ }^{I} V_{0} \\
{ }^{0} \dot{V}_{0} & \equiv{ }^{0} R_{I} \cdot \frac{\mathrm{d}^{I} V_{0}}{\mathrm{~d} t} & { }^{0} \dot{\omega}_{0} & \equiv{ }^{0} R_{I} \cdot \frac{\mathrm{d}^{I} \omega_{0}}{\mathrm{~d} t}
\end{aligned}
$$

where ${ }^{I} V_{0} \in \mathbb{R}^{3}$ and ${ }^{I} \omega_{0} \in \mathbb{R}^{3}$ are respectively the linear and angular velocity of the zero-frame with respect to the inertial frame. The rotation matrix ${ }^{0} R_{I} \in \mathbb{R}^{3 \times 3}$ expresses the orientation of the inertial frame with respect to the zero-frame. As the right foot moves, the matrix ${ }^{0} R_{I}$ is not constant. It can be shown that (11) implies:

$$
\begin{aligned}
& \frac{\mathrm{d}^{0} \dot{V}_{0}}{\mathrm{~d} t}={ }^{0} \dot{V}_{0}-{ }^{0} \omega_{0} \times{ }^{0} V_{0} \\
& \frac{\mathrm{d}^{0} \dot{\omega}_{0}}{\mathrm{~d} t}={ }^{0} \dot{\omega}_{0},
\end{aligned}
$$

as consequence the time derivative of $q_{v}$ is not $q_{a}$. Concerning the equation (10), $A \in \mathbb{R}^{20 \times 20}$ is the inertia matrix, $H \in \mathbb{R}^{20}$ is the vector including Coriolis, centrifugal and gravitational terms, $\Gamma \in \mathbb{R}^{14}$ is the control input, $D \in$ $\mathbb{R}^{20 \times 24}$, is the transposed of the Jacobian matrix relating the Cartesian velocities of the vertices of the foot with the joint velocities. The vector $F=\left[f_{1}^{T} \ldots f_{8}^{T}\right]^{T} \in \mathbb{R}^{24}$ contains all contact force vectors. Each contact force $f_{i}$ is a three dimensional vector containing the tangential forces $f_{i_{t}} \in \mathbb{R}^{2}$ and the normal force $f_{n} \in \mathbb{R}$

$$
f_{i}=\left[\begin{array}{c}
f_{i_{t}} \\
f_{i_{n}}
\end{array}\right], \quad f_{i_{t}}=\left[\begin{array}{l}
f_{i_{x}} \\
f_{i_{y}}
\end{array}\right] .
$$


Contact forces are subject to the following constraints:

$$
f_{i_{n}} \geq 0, \quad\left|f_{i_{t}}\right| \leq \mu f_{i_{n}} \quad i=1 \ldots 8
$$

Every contact point has an associated scalar constraint $\phi_{i}(q, \alpha) \geq 0$. Being $\phi_{i}(q, \alpha)$ the distance between the vertice $i$ of the foot and the ground. When $\phi_{i}(q, \alpha)>$ 0 , the vertice is not in contact with the ground and the corresponding $f_{i}$ is zero. Conversely, when $\phi_{i}(q, \alpha)=0$, the magnitude of the corresponding $f_{i}$ is not zero. Given $n_{c}\left(t_{k}\right)$, the set of contact points satisfying the condition $\phi_{i}(q, \alpha)=0$ at the instant time $t=t_{k}$, the term $D(q, \alpha) F$ of the equation (10) at such instant can be written in terms of the nonzero contact forces

$$
D(q, \alpha) F=\sum_{i \in n_{c}} D_{i}(q, \alpha) f_{i}
$$

$D_{i} \in \mathbb{R}^{20 \times 3}$ is composed by the three columns of $D$ corresponding to the forces $f_{i_{n}}, f_{i_{x}}$ and $f_{i_{y}}$. For example, if at a given instant the vertices $v_{1}$ and $v_{4}$ (see Figure 8) are contact with the ground, then $n_{c}=\{1,4\}$. In such case $D_{1}$ is composed of the columns of the matrix $D$ corresponding to the forces $f_{1_{n}}, f_{1_{x}}, f_{1_{y}}$, and $D_{4}$ of those corresponding to $f_{4_{n}}, f_{4_{x}}, f_{4_{y}}$. The equation (13) can be rewritten in a compact form as:

$$
D(q, \alpha) F=D_{c}(q, \alpha) F_{c}
$$

$D_{c}$ is composed by the matrix $D_{i}, i \in n_{c}$, and $F_{c}$ by the vectors $f_{i}, i \in n_{c}$. Now consider $P=\left[p_{1}^{T} \ldots p_{8}^{T}\right]^{T} \in \mathbb{R}^{24}$, the vector containing the Cartesian position of the vertices of the foot in the inertial frame. With $p_{i}(q)=\left[p_{i_{x}}, p_{i_{y}}, p_{i_{z}}\right]^{T} \in$ $\mathbb{R}^{3}$. The relationship between $\dot{P}$ and $q_{v}$ is given by the Jacobian matrix $J=D^{T}$ :

$$
\dot{P}=D^{T} q_{v}
$$

Taking the derivative of the kinematic equation above, the Cartesian accelerations $\ddot{P}$ can be exprimed as a linear function of $F$,

$$
\begin{aligned}
\ddot{P} & =D^{T} q_{a}+B \\
& =\left[D^{T} A^{-1} D\right] \cdot F+\left[D^{T} A^{-1}\left(\Gamma_{e}-H\right)+B\right]
\end{aligned}
$$

with

$$
B \equiv-D^{T}\left[\begin{array}{c}
{ }^{0} \omega_{0} \times{ }^{0} V_{0} \\
0 \\
0
\end{array}\right]+\dot{D}^{T} q_{v}
$$

If instead of the eight Cartesian positions, we consider only the Cartesian positions of the vertices in contact with the ground $\left(P_{c}(q)=\left[p_{i}^{T}\right]^{T}, i \in n_{c}\right)$, the equation (15) becomes:

$$
\ddot{P}_{c}=\left[D_{c}^{T} A^{-1} D_{c}\right] \cdot F_{c}+\left[D_{c}^{T} A^{-1}\left(\Gamma_{e}-H\right)+B_{c}\right]
$$

with

$$
B_{c} \equiv-D_{c}^{T}\left[\begin{array}{c}
{ }^{0} \omega_{0} \times{ }^{0} V_{0} \\
0 \\
0
\end{array}\right]+\dot{D}_{c}^{T} q_{v}
$$

The main point of our approach is to consider that $\ddot{P}_{c}$ is imposed by the contact model and then solve for $F_{c}$ from (16). The accelerations given by the contact model will

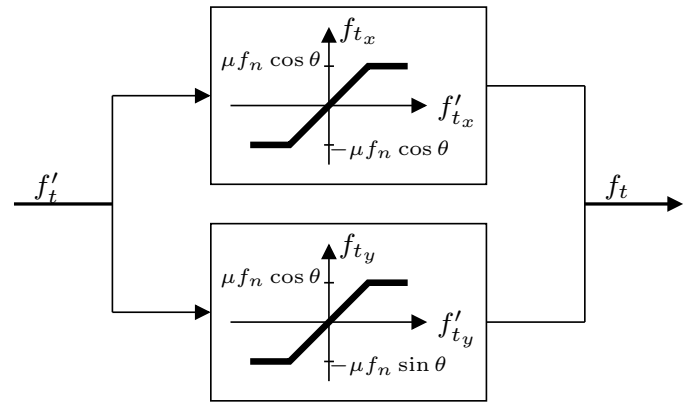

Fig. 9. Nonlinearities used to get tangential contact forces inside of the friction cone.

be denoted by $\ddot{P}^{d}$. The component $\ddot{p}_{i}^{d}$ of the vector $\ddot{P}^{d}$ is the required acceleration to asymptotically stops the motion of the contact point $i\left(\dot{p}_{i} \rightarrow 0\right)$ and removes its interpenetration $\left(p_{i_{z}} \rightarrow 0\right)$

$$
\ddot{p}_{i}^{d}=-k_{p}\left[\begin{array}{c}
0 \\
0 \\
p_{i_{z}}
\end{array}\right]-k_{v}\left[\begin{array}{c}
\dot{p}_{i_{x}} \\
\dot{p}_{i_{y}} \\
\min \left(0, \dot{p}_{i_{z}}\right)
\end{array}\right], \quad i \in n_{c}
$$

Once the desired accelerations are defined, the following step is to compute the required $F_{c}^{\prime}$ to obtain $\ddot{P}=\ddot{P}^{d}$

$$
\left[D_{c}^{T} A^{-1} D_{c}\right] \cdot F_{c}^{\prime}=\ddot{P}^{d}-\left[D_{c}^{T} A^{-1}\left(\Gamma_{e}-H\right)+B_{c}\right]
$$

The system of linear equations (18) may have an infinity of solutions. For example, in a bipedal robot, when the four vertices of the foot are in contact with the ground during a single support phase, the contact forces cannot be uniquely determined. In such cases, the solution with minimum Euclidean norm is computed using the MoorePenrose pseudo-inverse. Once $F_{c}^{\prime}$ is computed, the following step consists to build the vector $F^{\prime}$ composed of $F_{c}^{\prime}$ and zeros. After that, saturations are applied to $F^{\prime}$ in order to obtain contact force vector $F$ satisfying (12). The following saturation function assures a nonnegative normal contact force:

$$
f_{i_{n}}=\max \left(0, f_{i_{n}}^{\prime}\right)
$$

As the maximal value of $\left|f_{i_{t}}\right|$ is $\mu f_{i_{n}}$, the maximal values for $\left|f_{i_{x}}\right|$ and $\left|f_{i_{y}}\right|$ are respectively $\mu f_{i_{n}} \cos \theta$ and $\mu f_{i_{n}} \sin \theta$. With $\theta=\operatorname{atan}\left(f_{i_{y}}^{\prime}, f_{i_{x}}^{\prime}\right)$. Thus, the components of the tangential force vector are computed using the procedure depicted in Figure (9). This procedure can be exprimed mathematically as:

$$
\begin{aligned}
& f_{i_{x}}=\operatorname{sign}\left(f_{i_{x}}^{\prime}\right) \cdot \min \left(\left|f_{i_{x}}^{\prime}\right|, \mu f_{i_{n}} \cos \theta\right) \\
& f_{i_{y}}=\operatorname{sign}\left(f_{i_{y}}^{\prime}\right) \cdot \min \left(\left|f_{i_{y}}^{\prime}\right|, \mu f_{i_{n}} \sin \theta\right)
\end{aligned}
$$

As it can be seen from this equation, the transition between dynamic and static friction does not produce discontinuous tangential forces and it does not require a priori defined velocity thresholds. If $F_{c}^{\prime}$ satisfies the physical constraints (12), then $\ddot{P}_{c}=\ddot{P}_{c}^{d}$. In such case, contact forces and accelerations are decoupled. In this approach, inspired from input-output feedback linearization, parameter selection for the contact 


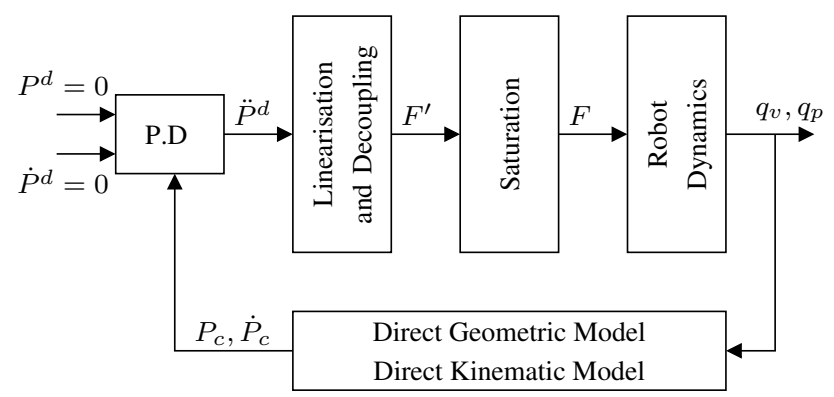

Fig. 10. Contact force computation for the bipedal robot described by (10).

model is independent on the number of points in contact with the ground and the parameters of the robot (i.e mass, inertias). The contact force computation for the system (10) is described in Figure 10.

\section{NUMERICAL TESTS}

The procedure described in this paper for contact force computation has been applied to the simulation of a walking gait consisting of five steps. The selected parameters for the Equation (17) were $k_{p}=500$ and $k_{v}=250$. In Figure 11 the distances between the vertices of the foot and the ground are traced as a function of the time. In Figure 12, these distances are traced when the right foot is in contact with the ground after its first impact. Figure 13 shows the corresponding normal contact forces.
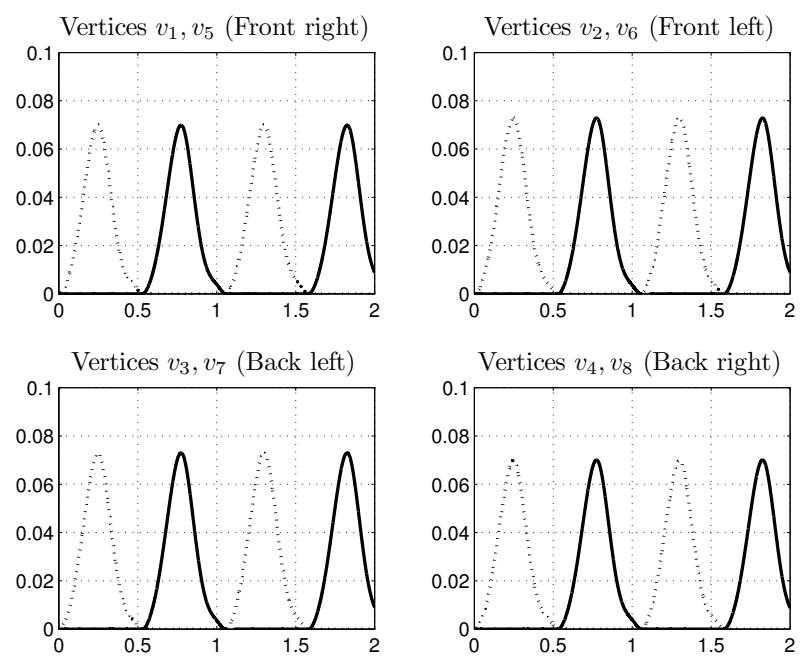

Fig. 11. Vertical position of the feet. Height $(\mathrm{m})$ versus time $(\mathrm{sec})$ for a walking gait consisting of four steps. Right foot (solid line), Left foot (dotted line)

\section{Contact stability}

In this section the numerical stability of the contact between the foot and the ground will be introduced. It has important consequences on normal forces computation. In the absence of external forces, the contact between an object and the
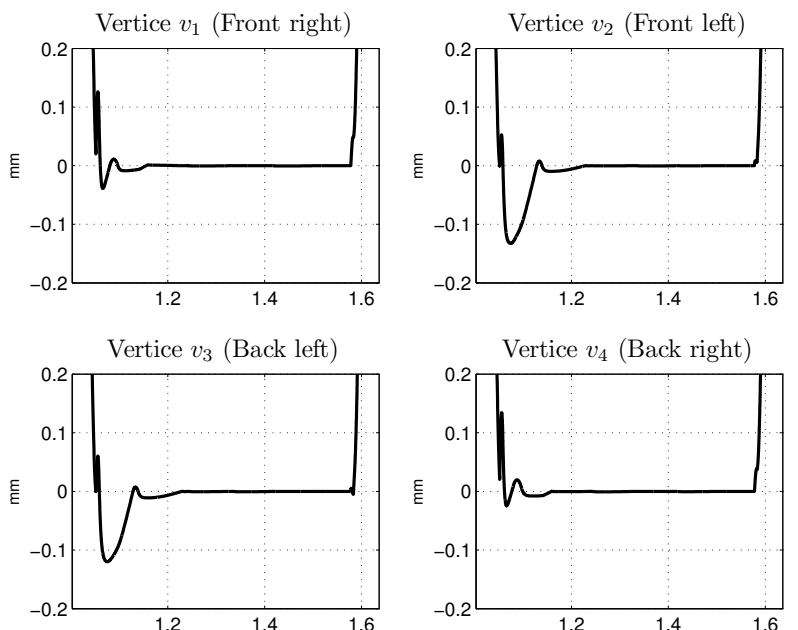

Fig. 12. Vertical position of the feet around zero height. Height $(\mathrm{mm})$ versus time $(\mathrm{sec})$ for the vertices of the right foot after its first impact.
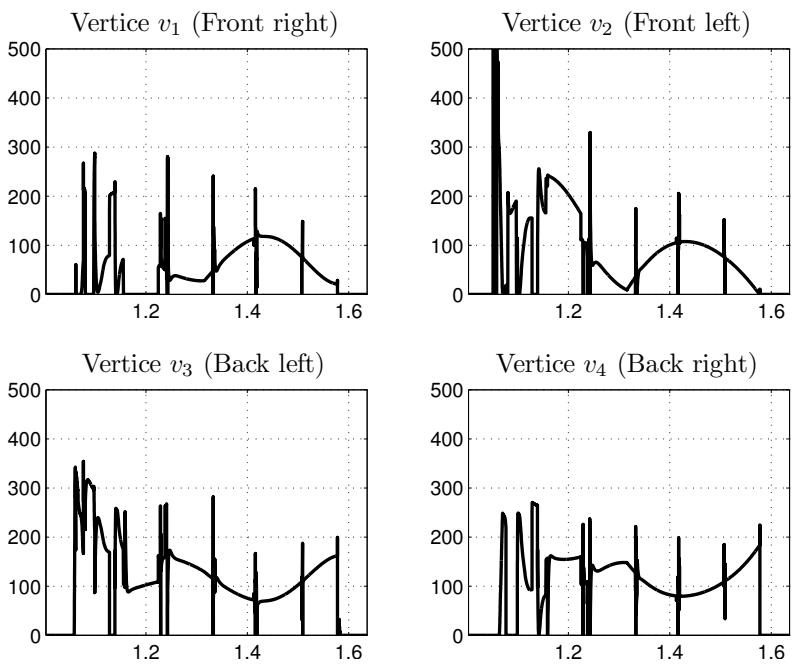

Fig. 13. Normal contact forces. Normal contact forces $(N)$ versus time (sec). These forces correspond to the simulations results presented in the Figure 12.

ground is said in equilibrium if after a given instant $T_{c}$, the normal force exerted by the ground is equal to the weight of the object. In such case, the application of a penaltybased approach leads to $p_{z}=\dot{p}_{z}=\ddot{p}_{z}=0, \forall t>T_{c}$. If an external force is added to the normal force exerted by the body on the ground, the equilibrium is broken and interpenetration immediately appears. However, after a transient time, position, velocity and acceleration return to zero and the normal force converges toward a new steady state value. If the external force mentioned above disappears, the contact is broken and a positive distance appears. As consequence, the normal contact force becomes zero. As no opposition to the gravity force exists, the object will be again in contact with the ground after a short instant generating a impact. These impacts are not physical ones, they occur only in simulation. For this reason, thereafter they will be called false 
impacts. This phenomena occurs in bipedal robot simulation. During the single support phases, the foot in contact with the ground is subject to a time varying forces generated by the motion of the other bodies. As we can see from Figure 13, false impacts produce discontinuities (vertical lines) in the computed normal forces. Contact stability can be improved if steady state interpenetration is allowed. Instead of converge towards $p_{i_{z}}=0$, the Equation (17) can be modified for obtaining convergence towards $p_{i_{z}}=\epsilon$.

$$
\ddot{p}_{i}^{d}=k_{p}\left[\begin{array}{c}
0 \\
0 \\
\epsilon-p_{i_{z}}
\end{array}\right]-k_{v}\left[\begin{array}{c}
\dot{p}_{i_{x}} \\
\dot{p}_{i_{y}} \\
\min \left(0, \dot{p}_{i_{z}}\right)
\end{array}\right], \quad i \in n_{c}
$$

The normal contact forces corresponding to $\epsilon=1 \times 10^{-6} \mathrm{~m}$ and the same values of $k_{p}$ and $k_{v}\left(k_{p}=500, k_{v}=250\right)$ are showed in Figure 14.
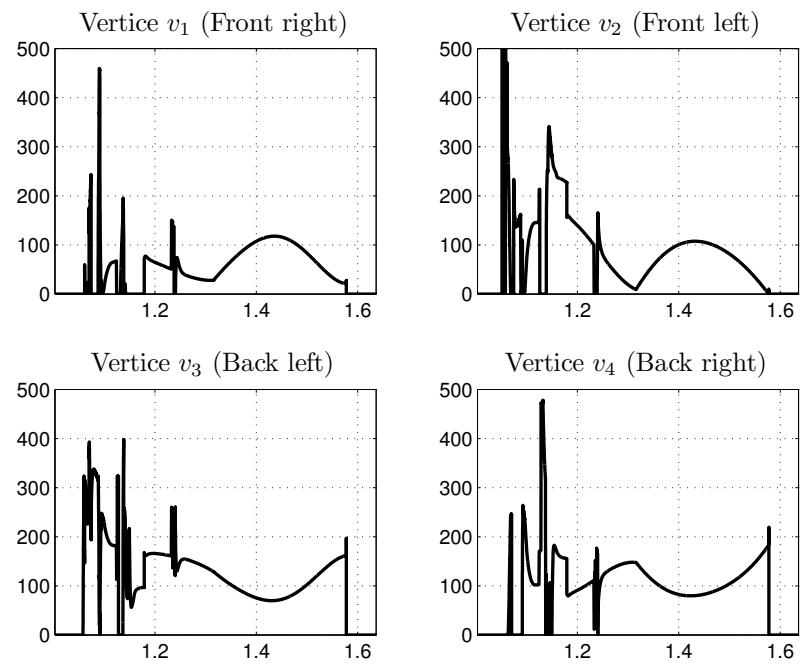

Fig. 14. Normal contact forces. Normal contact forces $(N)$ versus time (sec) when steady-state interpenetration is allowed. These results must be compared of those presented in Figure 13.

\section{CONCLUSION}

In this paper we have presented a new penalty approach based on input-output feedback linearization. This idea coming from control theory, allows to treat multiple simultaneous contacts independently. Another advantage is parameters of the contact model are independent of the parameters of the system. The computation of contact forces is composed of two steps. First, the required forces to avoid interpenetration and stop the motion of the contact points are computed. Second, nonlinearities are applied to these forces in order to satisfy physical constraints. The proposition was successfully applied to the simulation of a 3D bipedal walking robot. A simple solution was proposed to improve the numerical stability of the contacts between the foot and the ground.

\section{REFERENCES}

[1] M. Anitescu and F. Potra, "A time-stepping method for stiff multibody dynamics with contact and friction," International Journal for Numerical Methods in Engineering, vol. 55, no. 7, pp. 753-784, 2002.
[2] T. Buschmann, S. Lohmeier, H. Ulbrich, and F. Pfeiffer, "Dynamics simulation for a biped robot: modeling and experimental verification," in Proceedings of the IEEE International Conference on Robotics and Automation, Orlando, Florida, May 2006, pp. 2673-2678.

[3] J. Chardonnet, S. Miossec, A. Kheddar, H. Arisumi, H. Hirukawa, F. Pierrot, and K. Yokoi, "Dynamic simulator for humanoids using constraint-based method with static friction," in IEEE International Conference on Robotics and Biomimetics, Kunming, China, 2006, pp. 1366-1371.

[4] M. Cline and D. Pai, "Post-stabilization for rigid body simulation with contact and constraints," in IEEE International Conference on Robotics and Automation, vol. 3, Sept 2003, pp. 3744-3751.

[5] E. Drumwright, "A fast and stable penalty method for rigid body simulation," IEEE Transactions on Visualization and Computer Graphics, vol. 14, no. 1, pp. 231-240, 2008.

[6] J. G. Hale, B. Hohl, and E. M. Moraud, "Robot simulation, collisions and contacts," in IEEE/RSJ International Conference on Intelligent Robots and Systems, Nice, France, Sept 2008.

[7] H. Hirukawa, F. Kanehiro, S. Kajita, K. Fujiwara, K. Yokoi, K. Kaneko, and K. Harada, "Experimental evaluation of the dynamic simulation of biped walking of humanoid robots," in Proceedings of the IEEE International Conference on Robotics and Automation, vol. 2, Sept 2003, pp. 1640-1645.

[8] Y. Hwang, E. Inohira, A. Konno, and M. Uchiyama, "An order $n$ dynamic simulator for a humanoid robot with a virtual spring-damper contact model," in Proceedings of the IEEE International Conference on Robotics and Automation, vol. 2, Sept 2003, pp. 1640-1645.

[9] T. Liu and M. Wang, "Computation of three-dimensional rigid-body dynamics with multiple unilateral contacts using time-stepping and Gauss-Seidel methods," IEEE Transactions on Automation Science and Engineering, vol. 2, no. 1, pp. 19-31, 2005.

[10] D. Marhefka and D. Orin, "Simulation of contact using a nonlinear damping model," in Proceedings of the IEEE International Conference on Robotics and Automation, vol. 2, April 1996, pp. 1662-1668.

[11] D. C. Ruspini and O. Khatib, "Collision/contact models for the dynamic simulation of complex environments," in 9th International Symposium of Robotics Research, Snowbird - USA, 1997, pp. 185195.

[12] D. Stewart and J. Trinkle, "An implicit time-stepping scheme for rigid body dynamics with coulomb friction," in Proceedings of the IEEE International Conference on Robotics and Automation, vol. 1, 2000, pp. 162-169.

[13] D. E. Stewart, "Convergence of a time-stepping scheme for rigid-body dynamics and resolution of painlevé's problem," Archive for Rational Mechanics and Analysis, vol. 145, no. 3, pp. 215-260, 1998.

[14] —, "Rigid-body dynamics with friction and impact," SIAM Review, vol. 42, no. 1, pp. 3-39, 2000.

[15] K. Yamane and Y. Nakamura, "Dynamic filter - concept and implementation of online motion generator for human figures," IEEE Transactions on Robotics and Automation, vol. 19, no. 3, pp. 421432, 2003.

[16] - "Stable penalty-based model of frictional contacts," in IEEE International Conference on Robotics and Automation, Orlando, Florida, May 2006, pp. 1904-1909. 